\title{
Moon Formation: Water and Life on the Moon
}

\author{
N. Chandra Wickramasinghe ${ }^{1,2,3}$, Gensuke Tokoro ${ }^{3}$ and Edward J. Steele ${ }^{4}$ \\ ${ }^{1}$ Buckingham Centre for Astrobiology, University of Buckingham, UK \\ ${ }^{2}$ Centre for Astrobiology, University of Ruhuna, Matara, Sri Lanka \\ ${ }^{3}$ Institute for the Study of Panspermia and Astroeconomics, Gifu, Japan \\ ${ }^{4}$ CY O'Connor ERADE Village Foundation, Piara Waters,WA, Australia \\ Email: ncwick@gmail.com
}

\begin{abstract}
A new theory of the formation of the Moon predominantly from Earth material, combined with the recent discovery of surface water reopens the possibility that microbial life might exist close to the lunar surface.
\end{abstract}

Keywords: Moon formation, water on the moon, bacteria, panspermia.

The theory of the formation of the Moon that has prevailed for several decades involves the impact of a giant Mars-sized body on the Earth 4.5 billion years ago. The shattered debris of this impactor, along with a much smaller contribution from Earth-material, orbited Earth and subsequently coalesced to form the Moon. On this model the mineral composition of the Moon was to be understood in terms of a predominant contribution from the impactor itself, with only a minor contribution from the Earth's surface material.

Recent studies by Mastrobuono-Battisti et al (2015) [2] have cast doubt on this giant impact theory, however. The analysis of lunar meteorites and moon rocks returned by the Apollo missions has shown very similar stable oxygen isotope compositions $\left(\Delta^{17} \mathrm{O}\right.$ values $)$ to the Earth's mantle material. This suggests a model in which a large number of small impacts led to the vaporization and ejection of material from the Earth's crust that first orbited the Earth and later condensed and coalesced to form the Moon. The timing of small body impacts on the Earth that triggered the process could arguably be coincident with the Hadean geological epoch at 4.1-4.2 billion years ago. This was a period of frequent comet and asteroid impacts and interestingly the time of the oldest evidence of terrestrial microbial life. Detrital zircons older than 4.1Ga discovered in rocks belonging to a geological outcrop in the Jack Hills region of Western Australia have recently been found to contain micron-sized graphite spheres with an isotopic signature of biogenic carbon (Bell et al, 2015) [1]. The graphite spheres are plausibly relics of microbiology that was established on Earth at this time.

This raises the possibility that Earth microbes may have been transferred to the Moon in a viable form as it aggregated from Earth-rocks 4.1-4.2 Ga ago. The possibility that microbes lodged in surface ejecta survived transport and took refuge in lunar habitats is ruled out only if the conditions on the newly formed Moon were irretrievably hostile to biology. A total lack of water could be one such constraint. Although studies of modern lunar material returned in the Apollo missions indicated a total absence of water, new studies carried out with instruments aboard the Indian spacecraft Chandrayan 1 have shown the clear evidence of water particularly near the poles (Sridharan et al, 2010 [5]). The amounts of water detected at the surface are still very small $(<0.1 \%)$ but greater quantities could be present at depths of several metres.

In its day-night cycle, as the Moon rotates on its axis with a period of 27 days, the sunlit surface can reach a temperature of $120{ }^{\circ} \mathrm{C}$ at the equator, while dark side of the moon can have temperatures dipping to $-150{ }^{\circ} \mathrm{C}$. Lower temperatures apply for higher latitudes and in the bottom of craters. Daynight temperature variations will, however, be significantly damped several metres below the surface due to the low thermal conductivity of overlying material. Conditions conductive to the presence of liquid water or even viable microorganisms cannot be excluded at appropriate depth in the lunar regolith.

In 1961 Carl Sagan argued that at a depth of less than 50cm some locations on the Moon could have stable temperatures between 0 and $70{ }^{\circ} \mathrm{C}$, citing microwave radiation data (Piddington and Minnett, 
1949) [3] that was consistent with this result. (https://science.nasa.gov/science-news/science-at-nasa/ 2010/12jul_rabbithole/)

As far as we are aware more recent data have not vitiated this claim (https://science.nasa.gov/ science-news/science-at-nasa/2010/12jul_rabbithole/). It is therefore reasonable to assume that stable bio-friendly conditions conducive to the survival of microbial life still prevail metres below the surface of the Moon. There is also no evidence that contradicts the possibility that primordial microbes that were present on the Earth during the Hadean epoch (Bell et al 2015) [1] were transferred to the Moon during the last phases of its formation 4.1 billion years ago. In conclusion we consider it premature to write off our nearest neighbour as a dead and lifeless satellite.

\section{References}

1. Bell EA, Boehnke P, Harrison T et al (2015). Potentially biogenic carbon preserved in a 4.1 billion-year-old zircon, PNAS, www.pnas.org/cgi/doi/10.1073/pnas.1517557112.

2. Mastrobuono-Battisi A, Perets HB \& Raymond SN (2015). A primordial origin for the compositional similarity between the Earth and Moon, Nature, 520, 212-215.

3. Piddington, JH and Minnett, HC, 1949, Microwave Thermal Radiation from the Moon, Australian J. Sci. Res., A, 2: 63 .

4. Sagan, C., 1961. Organic Matter and the Moon, Report of Panel on Extra-Terrestrial Life for the Armed Forces$N R$ http://www.nap.edu/catalog.php?record_id=18476

5. Sridharan, R. et al (2010). Direct detection of water $\mathrm{H} 2 \mathrm{O}$ in sunlit ambience from CHACE on MIP of Chadrayaan 1. Planet Sp.Sci., 58, 947-950. 